\title{
POLYRADICULONEURITIS AS AN INITIAL MANIFESTATION OF BRONCHIAL CARCINOMA
}

\author{
Tommaso Claudio Mineo, MD, Fausto Biancari, MD, and Carlo Umberto Casciani, MD, Rome, Italy
}

Lung cancer, particularly small-cell lung cancer, may cause paraneoplastic neurologic syndromes. ${ }^{1-3}$ To our knowledge, at least four cases of paraneoplastic polyradiculoneuritis in association with this malignant tumor have been described. ${ }^{1,4}$ We report a case of polyradiculoneuritis as the presenting manifestation of poorly differentiated epidermoid carcinoma of the lung.

A 73-year-old male patient who had been smoking for a long time presented with asthenia, cough, paraesthesias in the toes, and bilateral weakness of the legs that made walking difficult. Symptoms had started 5 days earlier. Despite a recent weight loss, his general condition was good. Neurologic examination revealed areflexia, minimal loss of sensation in both legs, and aching of the large muscles of the thighs on palpation. Blood tests revealed leukocytosis $(20,000 / \mathrm{ml})$. Roentgenograms, computed tomographic scans, and magnetic resonance imaging of the chest showed a right hilar mass stenosing the right main stem bronchus. This observation was confirmed at bronchoscopy. Neither cerebral metastases nor abnormalities of the rachis were found. Total body bone scanning showed no abnormalities. Cytologic examination of the sputum diagnosed poorly differentiated epidermoid carcinoma of the lung and mediastinoscopy revealed high ipsilateral metastatic lymph nodes, which ruled out further surgical management. Examination of cerebrospinal fluid that showed normal pressure, no cells, a protein level of $0.90 \mathrm{gm} / \mathrm{L}$, and abnormal electrophysiologic findings indicated a diagnosis of polyradiculoneuritis. The patient was treated with intravenous methylprednisolone $500 \mathrm{mg}$ daily for 7 days without improvement of the symptoms, and weakness of facial muscles, of the arms, dysphagia symptoms, and paraplegia developed. He was treated with exchange $250 \mathrm{ml}$ of plasma per kilogram of body weight in five sessions within 10 days, and symptoms lessened rapidly. Eighteen days later, neurologic symptoms returned and respiratory failure appeared to require mechanical ventilation. He was again treated with plasma exchange without improvement of symptoms; 25 days

From the Department of Thoracic Surgery, University Tor Vergata of Rome, Rome, Italy.

J Thorac CARdIovasc Surg 1995;109:1254

Copyright $(\mathcal{C} 1995$ by Mosby-Year Book, Inc.

$0022-5223 / 95 \$ 3.00+0 \quad \mathbf{1 2 / 8 / 6 0 6 7 6}$ later he died of pulmonary sepsis. At autopsy, neither cerebral nor meningeal signs of metastastic involvement were found. Histologic examination of peripheral nerves showed lymphocytes and macrophages surrounding endoneural vessels and causing adjacent demyelination. These observations excluded metastatic meningoradiculitis ${ }^{4}$ and suggested a paraneoplastic origin of the neurologic syndrome. ${ }^{1}$ Conduction block may be produced by neoplastic release of humoral factors that disrupt electrical nerve conduction. Lymphocytic T-cell mechanism may play a part in the humoral and cell-mediated process of inflammation and demyelination as an aberrant response to an immunologic stimulus.

We conclude that this rare paraneoplastic neurologic syndrome is correlated with a rapid onset and a progressive and fatal course unresponsive to corticosteroids and plasma exchange. After the successful infusion of immune globulin in several immune-mediated diseases, the randomized Dutch trial has demonstrated the efficacy of this treatment in acute Guillan-Barré syndrome. ${ }^{5}$ However, its role in the management of paraneoplastic polyradiculoneuritis must be established.

\section{REFERENCES}

1. M'Raihi ML, Boukhris R, Zegaya M. Polyradiculonèvrite rèvèlatrice d'un cancer bronchopulmonaire. Rapport d'un cas et revue brève de la littèrature. Rev Mal Respir 1989;6:465-7.

2. Zenone T. Autoimmunity and cancer: paraneoplastic neurological syndromes associated with small cell cancer. Bull Cancer 1992;79:837-53.

3. Schiller JH, Jones JC. Paraneoplastic syndromes associated with lung cancer. Curr Opin Oncol 1993;5:33542.

4. Pissas A, Perret J, De Rougemont J, Vrousos C, Rachail $M$, Schaerer R. La mèningoradiculite mètastatique: un mode de rèvèlation exceptionnel de cancer gastrique. Revue de la littèrature a propos d'un cas. Semin Hop Paris 1981;57:1185-91.

5. van der Mechè FGA, Schmitz PIM, The Dutch Guillan-Barrè Study Group. A randomized trial comparing intravenous immune globulin and plasma exchange in Guillan-Barrè syndrome. N Engl J Med 1992;326:1123-9. 